\title{
Elevated Histamine Model: A Protocol for an ex vivo model for in vitro study of histamine effect on placenta version 2
}

\section{Obed Brew, Mark HF Sullivan}

\begin{abstract}
Maternal plasma histamine levels in normal pregnancy are generally similar to those in non-pregnant women during the first trimester, and usually decline gradually during the $2^{\text {nd }}$ and $3^{\text {rd }}$ trimesters (Brew and Sullivan, 2006). In some complications of pregnancy such as hyperemesis gravidarum (HG), spontaneous abortion (SA), pre-term labour (PL) and Pre-eclampsia (PE) histamine levels increase as pregnancy proceeds (Southren et al., 1966; Achari, Achari and Rao, 1971; Beaven et al., 1975), and the elevated levels of histamine may directly cause some of the features of these disorders (Brew and Sullivan, 2006).
\end{abstract}

Placenta is a major source of histamine, but it also releases active diamine amine oxidase (DAO; EC 1.4.3.22) into the maternal circulation, which metabolises histamine (Kapeller-Adler, 1944; Gunther and Glick, 1967; Semeniuchenko, 1975; Granerus, Gillbrand and Wetterqvist, 1977; Purcell and Hanahoe, 1991; Brew, Lakasing and Sullivan, 2007). This breaks down the histamine released from the placenta in normal pregnancy, leading to the limited changes in maternal blood histamine. Clinically, the deported placental DAO activity in maternal blood increases a 1000 fold during normal pregnancy (Southren et al., 1966).

The DAO activity rises exponentially in the first 24 weeks of normal gestation and plateaus thereafter to form a normal histamine-DAO-axis (nHDA) (Southren et al., 1966; Beaven et al., 1975; Dubois et al., 1977) (Ahlmark, 1944; Southren et al., 1966; Gunther and Glick, 1967; Weingold and Southren, 1968; Tufvesson, 1978; Beaven et al., 1975; Dubois et al., 1977). In contrast, the spontaneous exponential rise of DAO activity is abrogated from gestational week 8 onwards thus, leading to a defective Histamine-DAO-Axis (dHDA) in pregnancies that present with elevated maternal blood histamine (Southren et al., 1966; Achari, Achari and Rao, 1971; Beaven et al., 1975; Legge and Duff, 1981). Ex-vivo defective Histamine-DAO-Axis also referred to Elevated Histamine Model (EHM) was developed to mimic in vivo dHDA to study effects of histamine in human placenta. The EHM was developed by creating in vitro culture model to represent normal placentae with nHDA and complicated placentae with dHDA. In the nHDA samples, the endogenous DAO activity is maintained to eliminate endogenous production of histamine, while DAO activity is blocked with aminoguanidine in the dHDA samples to allow histamine levels to elevate during the treatment period.

Aminoguanidine is a specific inhibitor of DAO enzyme activity (Tamura et al., 1989).

Citation: Obed Brew,Mark HF SullivanElevated Histamine Model: A Protocol for an ex vivo model for in vitro study of histamine effect on placenta. protocols.io

dx.doi.org/10.17504/protocols.io.jigckbw

Published: 23 Aug 2017

\section{Guidelines}

Note:

Syncytiotrophoblast degeneration starts within 24 hours of explant culture and peaks by 48 hours. Syncytiotrophoblast regeneration is established by120 hours of culture. Therefore culture explants 
for 5 days and apply elevated histamine treatment thereafter for a further 24 hours.

Explants culltured for 48 hours produced extremely poor quality RNA for high throughput downstream analysis.

Micro explants incubated at the bottom of the well was associated with decreased explant viability and poor RNA quality.

\section{Protocol}

Human Placental Tissue Collection and Preparation

1. Obtain ethics permission and patient consent prior to placental collection.

2. Record clinical characteristics of pregnancies for placentae to be studied as Table 1 (sample Table 1 available from http://www.elsevier.com/_data/promis_misc/YPLAC\%20Guide\%20for\%20authors\%20Table\%201.doc).

3. Collect from operating theatre term (38-39 weeks of gestation) placenta delivered by caesarean section from normal pregnancy.

4. Inspect the placenta and record evidence for gross abnormalities such as placental infarcts, excessive tears, necrosis, absent cotyledons, discolorations on the maternal and fetal surfaces, and anomalies in cord insertion.

5. Cut $2 \mathrm{~cm}^{3}$ of placental samples randomly from at least 3 different healthy looking sampling sites about $5 \mathrm{~cm}$ away from the umbilical cord with sterile sharp scissors.

6. Excise each sample to include an intact chorionic plate, intervillous space, basal plate and decidua.

7. Wash each placental sample in phosphate-buffered saline (PBS) solution (pH 7.4) containing $10 \%$ penicillin, streptomycin and L-glutamine (Sigma) to remove excess blood and blood clots.

8. Transport each washed sample in $150 \mathrm{~mL}$ sterile pots containing warm PBS with $10 \%$ penicillin, streptomycin and L-glutamine immediately to the laboratory.

9. Aseptically dissect micro explants ( $<50 \mathrm{mg}$ wet weight) of villous tissue with sterile scalpel from each sample.

10. Incubate the micro explant within 30 min of delivery.

\section{Standard Culture}

11. Place each fragmented micro explant on a mesh support in a 12-well culture plate containing $15 \mathrm{~mm}$ diameter Netwell inserts with $74 \mu \mathrm{m}$ polyester mesh bottoms attached to polystyrene inserts (Corning, UK). 
12. Add basic media: RPMI 1640 culture medium (Invitrogen, UK) supplemented with 2 mM L-glutamine, $10 \%$ fetal bovine serum, $100 \mathrm{IU} / \mathrm{ml}$ penicillin and $100 \mu \mathrm{g} / \mathrm{ml}$ streptomycin.

13. Incubate micro explants in basic media at the liquid-gas interface in $8 \%$ oxygen and $5 \% \mathrm{CO}_{2}$, at $37^{\circ} \mathrm{C}$ for 5 days. Note: culture at the bottom of the well was associated with decreased explant viability and poor RNA quality.

14. Replace culture media at days 2 and 4: place the media in a sterile container and expose to $8 \%$ oxygen tension for 2 hours before the medium change is done. Keep the time for the change of medium to the minimum (less than 5 minutes).

\section{Elevated Histamine Culture}

15. To create the elevated histamine model, repeat media change on day 5 for elevated histamine treatment:

1. Replace media for samples designated as dHDA samples with final media containing 100nM histamine in the presence of aminoguanidine $\left(10^{-4} \mathrm{M}\right.$ final concentration)

2. Replace media for samples designated as nHDA with final basic media without aminoguanidine or histamine.

3. Culture samples for further 24 hours

16. At the end of the 6 days culture period collect the explants and store immediately in RNAlater (Ambion) at $-80^{\circ} \mathrm{C}$.

17. Store pre-culture, $0 \mathrm{~h}$ control samples obtained from fresh placentae in RNAlater at $-80^{\circ} \mathrm{C}$ within 30 minutes of delivery. 